\title{
On the Phase Dependence of Double-Resonance Experiments in Rotational Spectroscopy Supplementary Material
}

David Schmitz ${ }^{1,2}$, V. Alvin Shubert ${ }^{1,2}$, David Patterson ${ }^{3}$, Anna Krin ${ }^{1,2}$, Melanie Schnell ${ }^{1,2 *}$ April 7, 2015

${ }^{1}$ Max-Planck-Institut für Struktur und Dynamik der Materie, Luruper Chaussee 149, D-22761 Hamburg, Germany

${ }^{2}$ Center for Free-Electron Laser Science, Luruper Chaussee 149, D-22761 Hamburg, Germany ${ }^{3}$ Harvard University, Department of Physics, Cambridge, USA 


\section{RF-MW double-resonance experiments of carvomenthenol}

In Figure 1, results for MW-RF double-resonance experiments on the lowest-energy conformer of 4carvomenthenol are shown. The respective rotational constants as obtained by broadband rotational spectroscopy are $\mathrm{A}=2236.4058(23) \mathrm{MHz}, \mathrm{B}=674.83573(65) \mathrm{MHz}$, and $\mathrm{C}=647.30432(55) \mathrm{MHz}$. In total, we identified three conformers in our molecular jet. We used a $1 \mu$ s chirp spanning 2-6 GHz to create coherence for a wide range of transitions and employed a $5 \mu$ s single frequency pulse to pump the $3_{12} \leftarrow 3_{13}$ transition at $165.2 \mathrm{MHz}$. We monitored the effect of the RF pumping on four different connected transitions $3_{12} \leftarrow 22_{11}, 3_{12} \leftarrow 22_{02}, 4_{13} \leftarrow 3_{12}$ and $4_{14} \leftarrow 3_{13}$ (top part of Figure 1). The corresponding microwave signal phases are displayed in the lower part of Figure 1 as a function of the RF pump frequency that was scanned in $10 \mathrm{kHz}$ steps. Note that the phase behavior for the $4_{13} \leftarrow 3_{12}$ microwave signal transition at $5342.89 \mathrm{MHz}$ is just opposite to the other ones. This difference again arises from the different energy level arrangement, which is progressive for the transition at 5342.89 $\mathrm{MHz}\left(J_{K_{a}^{\prime} K_{c}^{\prime}}^{\prime} \leftarrow J_{K_{a} K_{c}}=4_{13} \leftarrow 3_{12}\right)$ and regressive for the remaining ones. This observation is in line with the results observed for menthone and isomenthone illustrated in Figures 3 and 4 in the main article. Note that for the signal transition at $5232.75 \mathrm{MHz}\left(J_{K_{a}^{\prime} K_{c}^{\prime}}^{\prime} \leftarrow J_{K_{a} K_{c}}=4_{14} \leftarrow 3_{13}\right)$ the energy level arrangement is a regressive $V$-type one. As can be seen, it follows the same phase behavior as the regressive $\Lambda$-type arrangement.

\section{Three-level optical Bloch equations}

The density matrix for a three-level system is a three-by-three matrix with diagonal elements $\rho_{i i}(i=$ $a, b, c)$ describing the population of the i-th level, whereas the off-diagonal elements $\rho_{i j}(i \neq j=a, b, c)$ describe the coherences between the states $|i\rangle$ and $|j\rangle$. Relaxation terms are neglected, which is justified due to the long $T_{2}$ times of rotational coherences. The corresponding Hamiltonian of a double-resonance experiment is the sum of the unperturbed Hamiltonian $\hat{\mathcal{H}}_{0}$ and the interaction term $\hat{\mathcal{V}}$ describing the interaction with the electromagnetic field $\hat{\mathcal{H}}=\hat{\mathcal{H}}_{0}+\hat{\mathcal{V}}$. The time evolution of the density matrix is then expressed by the Liouville equation [1]:

$$
i \hbar \frac{\partial \hat{\rho}(t)}{\partial t}=[\hat{\mathcal{H}}(t), \hat{\rho}(t)]
$$

and the expectation value for the experimentally accessible polarization $P$ is given by:

$$
\langle\hat{P}\rangle=N \cdot \operatorname{Tr}\{\hat{\rho} \hat{\mu}\}
$$

where $N$ is the number of excited molecules and $\hat{\mu}$ is the transition dipole-moment operator. In the following we provide explicit expressions for the Hamiltonian $\hat{\mathcal{H}}_{\text {pro }}$ in the progressive regime and the corresponding optical Bloch equations. The Hamiltonians and the optical Bloch equations for the 

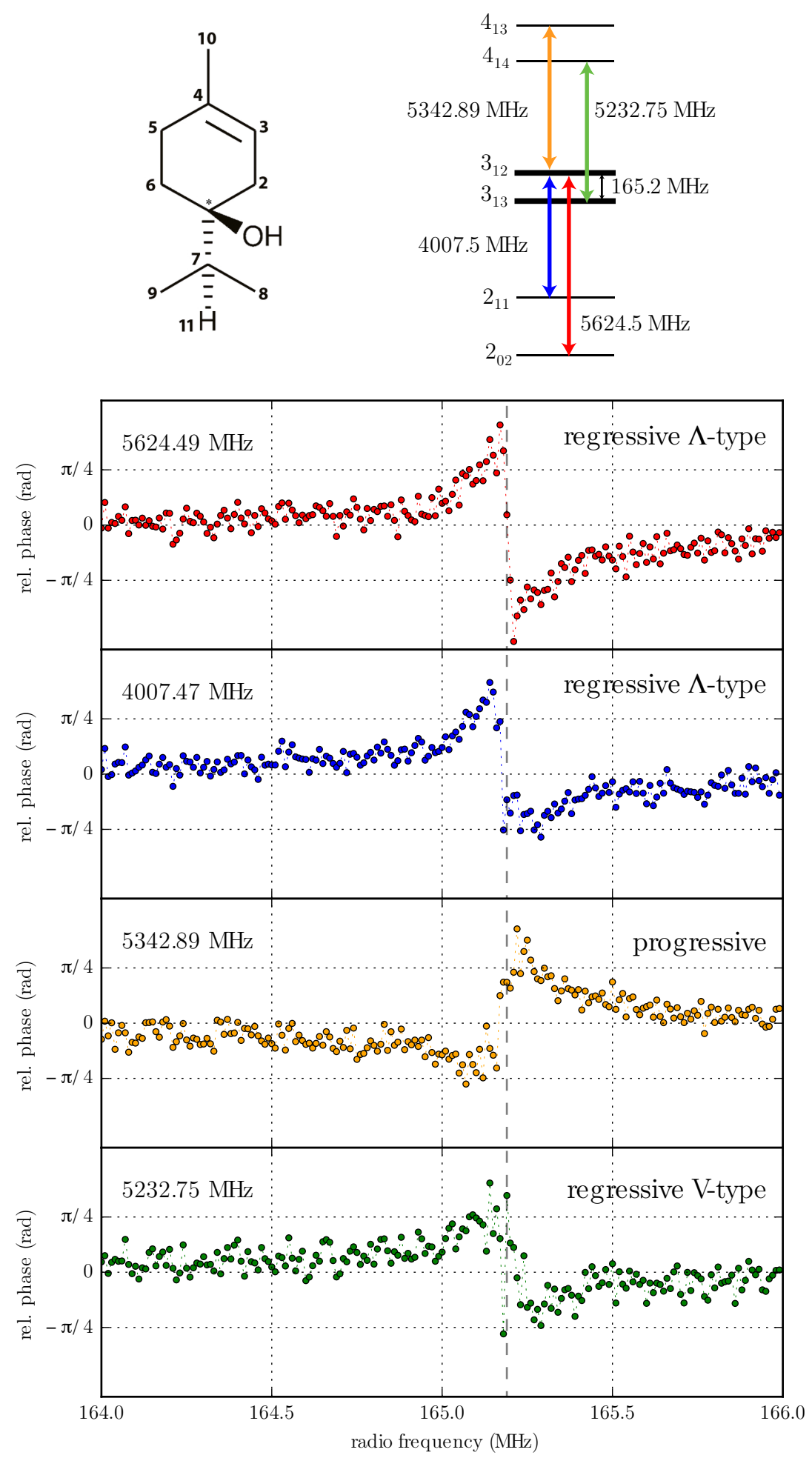

Figure 1: MW-RF double-resonance experiments for the lowest-energy conformer of 4-carvomenthenol. A scheme of the molecular structure as well as the corresponding energy level arrangement is given in upper part. The color code of the arrows included in the energy-level arrangement is resembled in the measurements of the phase dependence of the four different microwave signal transitions on the RF pump frequency involving the $3_{12} \leftarrow 3_{13}$ transition at $165.2 \mathrm{MHz}$. 
regressive regime ( $\Lambda$-type or $V$-type) are obtained in the same manner.

$$
\hat{\mathcal{H}}_{\text {pro }}=\left(\begin{array}{ccc}
E_{a} & -\frac{1}{2} \mu_{a b} \mathcal{E}_{a b}\left(e^{i \omega_{\mathrm{ab}} t}+e^{-i \omega_{\mathrm{ab}} t}\right) & 0 \\
-\frac{1}{2} \mu_{b a} \mathcal{E}_{b a}\left(e^{i \omega_{\mathrm{ab}} t}+e^{-i \omega_{\mathrm{ab}} t}\right) & E_{b} & -\frac{1}{2} \mu_{b c} \mathcal{E}_{b c}\left(e^{i \omega_{\mathrm{bc}} t}+e^{-i \omega_{\mathrm{bc}} t}\right) \\
0 & -\frac{1}{2} \mu_{c b} \mathcal{E}_{c b}\left(e^{i \omega_{\mathrm{bc}} t}+e^{-i \omega_{\mathrm{bc}} t}\right) & E_{c}
\end{array}\right)
$$

A few approximations have been applied to reduce the complexity. One is that the interaction volume is small compared to the wavelength by neglecting the dependence of the electromagnetic field on the wave vector k. Also the initial phases of both incoming waves were set to zero. Furthermore, the assumption is made that each of the two incoming fields, the MW and the RF field, are exclusively coupling to different transition dipole moment components $\mu_{i j}$ (with $i \neq j=a, b, c$ ). This assumption is valid for this work, because the transition frequencies are well separated and the polarization direction of the two incoming waves is perpendicular to each other in our experimental setup.

Set of the optical Bloch equations in the progressive regime:

$$
\begin{aligned}
i \hbar \frac{\partial \rho_{a a}}{\partial t} & =-\frac{\mathcal{E}_{a b}}{2}\left(\mu_{a b} \rho_{b a}-\mu_{a b}^{*} \rho_{a b}\right)\left(e^{i \omega_{a b} t}+e^{-i \omega_{a b} t}\right) \\
i \hbar \frac{\partial \rho_{b b}}{\partial t} & =\frac{\mathcal{E}_{a b}}{2}\left(\mu_{a b}^{*} \rho_{a b}-\mu_{a b} \rho_{b a}\right)\left(e^{i \omega_{a b} t}+e^{-i \omega_{a b} t}\right)+\frac{\mathcal{E}_{b c}}{2}\left(\mu_{b c} \rho_{c b}-\mu_{b c}^{*} \rho_{b c}\right)\left(e^{i \omega_{b c} t}+e^{-i \omega_{b c} t}\right) \\
i \hbar \frac{\partial \rho_{c c}}{\partial t} & =-\frac{\mathcal{E}_{b c}}{2}\left(\mu_{b c}^{*} \rho_{b c}-\mu_{b c} \rho_{c b}\right)\left(e^{i \omega_{b c} t}+e^{-i \omega_{b c} t}\right) \\
i \hbar \frac{\partial \rho_{a b}}{\partial t} & =-\hbar \omega_{0, a b} \rho_{a b}-\frac{\mathcal{E}_{a b} \mu_{a b}}{2}\left(\rho_{b b}-\rho_{a a}\right)\left(e^{i \omega_{a b} t}+e^{-i \omega_{a b} t}\right)+\frac{\mathcal{E}_{b c} \mu_{b c}^{*}}{2} \rho_{a c}\left(e^{i \omega_{b c} t}+e^{-i \omega_{b c} t}\right) \\
i \hbar \frac{\partial \rho_{a c}}{\partial t} & =-\hbar \omega_{0, a c} \rho_{a c}-\frac{\mathcal{E}_{a b} \mu_{a b}}{2} \rho_{b c}\left(e^{i \omega_{a b} t}+e^{-i \omega_{a b} t}\right)+\frac{\mathcal{E}_{b c} \mu_{b c}}{2} \rho_{a b}\left(e^{i \omega_{b c} t}+e^{-i \omega_{b c} t}\right) \\
i \hbar \frac{\partial \rho_{b a}}{\partial t} & =\hbar \omega_{0, a b} \rho_{b a}-\frac{\mathcal{E}_{a b} \mu_{a b}^{*}}{2}\left(\rho_{a a}-\rho_{b b}\right)\left(e^{i \omega_{a b} t}+e^{-i \omega_{a b} t}\right)-\frac{\mathcal{E}_{b c} \mu_{b c}}{2} \rho_{c a}\left(e^{i \omega_{b c} t}+e^{-i \omega_{b c} t}\right) \\
i \hbar \frac{\partial \rho_{b c}}{\partial t} & =-\hbar \omega_{0, b c} \rho_{b c}-\frac{\mathcal{E}_{a b} \mu_{a b}^{*}}{2} \rho_{a c}\left(e^{i \omega_{a b} t}+e^{-i \omega_{a b} t}\right)-\frac{\mathcal{E}_{b c} \mu_{b c}}{2}\left(\rho_{c c}-\rho_{b b}\right)\left(e^{i \omega_{b c} t}+e^{-i \omega_{b c} t}\right) \\
i \hbar \frac{\partial \rho_{c a}}{\partial t} & =\hbar \omega_{0, a c} \rho_{c a}+\frac{\mathcal{E}_{a b} \mu_{a b}^{*}}{2} \rho_{c b}\left(e^{i \omega_{a b} t}+e^{-i \omega_{a b} t}\right)-\frac{\mathcal{E}_{b c} \mu_{b c}^{*}}{2} \rho_{b a}\left(e^{i \omega_{b c} t}+e^{-i \omega_{b c} t}\right) \\
i \hbar \frac{\partial \rho_{c b}}{\partial t} & =\hbar \omega_{0, b c} \rho_{c b}+\frac{\mathcal{E}_{a b} \mu_{a b}}{2} \rho_{c a}\left(e^{i \omega_{a b} t}+e^{-i \omega_{a b} t}\right)-\frac{\mathcal{E}_{b c} \mu_{b c}^{*}}{2}\left(\rho_{b b}-\rho_{c c}\right)\left(e^{i \omega_{b c} t}+e^{-i \omega_{b c} t}\right)
\end{aligned}
$$

We simulated the intensity and the phase behavior using the respective set of optical Bloch equations in a two pulse scheme. The first pulse is a short and intense $\frac{\pi}{2}$-pulse at the resonance frequency of the $|a\rangle \rightarrow|b\rangle$ transition to create maximum $a b$-coherence. Note that in the experiments we used a chirp and excite both the $|a\rangle \rightarrow|b\rangle$ and the $|a\rangle \rightarrow|c\rangle$ transition. The second pulse used in the simulations is a $5 \mu$ sF pulse with the corresponding detuning from resonance (in agreement with the experiment). We adjusted the Rabi frequency $\Omega_{b c}$ to match the experimental results e.g. the intensity and the phase behavior for different detunings. For the initial populations of the states we assumed a Boltzmann distribution and employed the rotational energies provided by the SPCAT program. In addition we performed simulations to investigate the dependence of the strength of the RF pump pulse on the RFMW double-resonance experiments. Simulations of the amplitude and phase dependence are displayed 


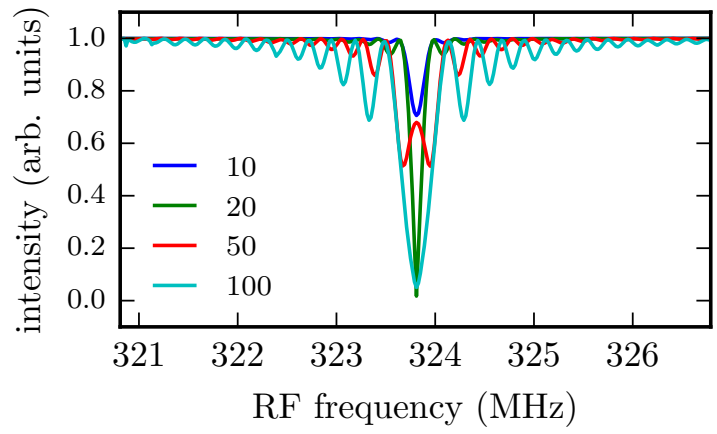

(a)

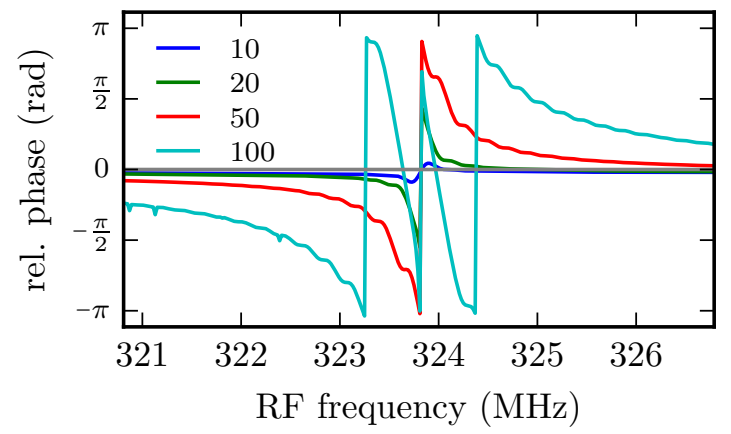

(b)

Figure 2: Simulations of the (a) amplitude and (b) phase dependence of the microwave signal transition on the RF pump detuning for four different RF Rabi frequencies. The Rabi frequencies are given in units of $D \frac{V}{m} / \hbar$.

in Figure 3. With increasing field strength, the decrease in signal amplitude becomes more pronounced until the RF-pulse hits $\pi$ and $2 \pi$ conditions (complete coherence transfer at resonance) at a Rabi frequency of $20 D \frac{V}{m} / \hbar$ or $100 D \frac{V}{m} / \hbar$, respectively. At a Rabi frequency of $50 D \frac{V}{m} / \hbar$ the pulse is between $\pi$ and $2 \pi$ conditions and hence the signal decrease is significantly less compared to a Rabi frequency of $20 \mathrm{D} \frac{\mathrm{V}}{\mathrm{m}} / \hbar$ or $100 D \frac{V}{m} / \hbar$. Because the oscillation of the populations depends on the generalized Rabi frequency, off-resonance peaks appear. However, if a transition is driven off-resonance no full population inversion or coherence transfer can be achieved.

As explained in the main article, the phase change reveals the shift of the energy levels due to the RF pumping. For higher field strength the phase change becomes more pronounced, because the shift of the energy levels is larger (due to the larger Rabi frequency $\Omega^{\prime}$ ).

\section{Autler-Townes doublet and field-strength dependence of the RF- MW double-resonance experiments}

As mentioned in the main article, we cannot observe the Autler-Townes doublet directly, but its influence on the phase of the MW signal. However, we performed simulations to illustrate the change in frequency and intensity of the Autler-Townes doublet as a function of the detuning for the example of menthone A. Figure 3a shows the spectrum of the $a b$-coherence for different detunings in a two-dimensional plot and also for a smaller set of detunings in a one-dimensional plot (Figure 3b). The simulations reveal an intensity ratio of:

$$
\frac{I_{c, N-1}}{I_{b, N}}=\tan ^{2}\left[\frac{1}{2} \cdot \arctan \left(-\frac{\Omega^{\prime}}{\Delta_{R F}}\right)\right]
$$

where $I_{c, N-1}$ and $I_{b, N}$ describe the intensities of the transitions between the state $|a, N\rangle$ and the dressed states evolving from the undressed states $|c, N-1\rangle$ and $|b, N\rangle$, respectively. 


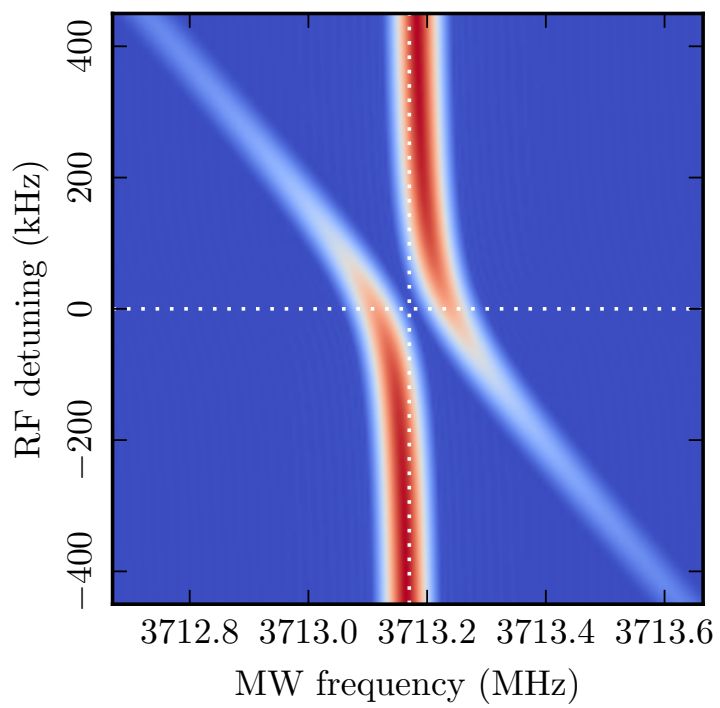

(a)

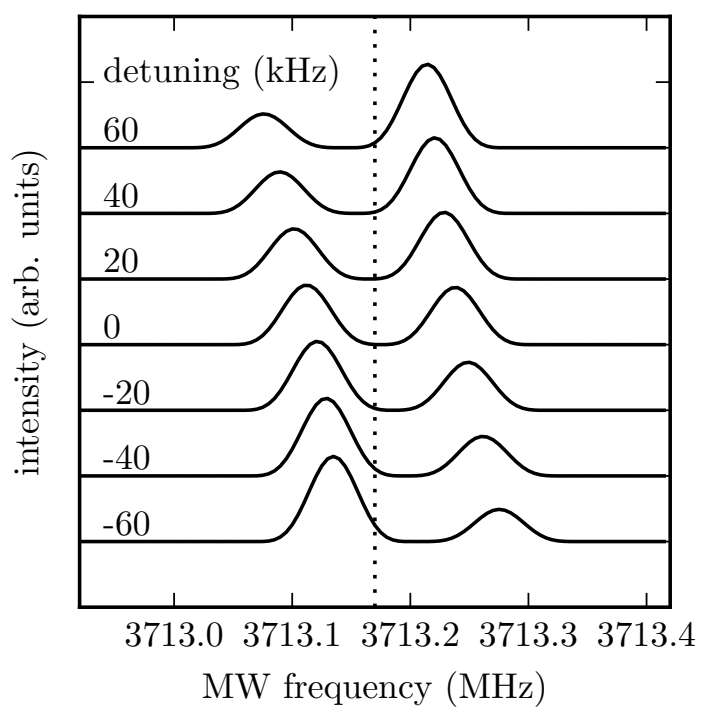

(b)

Figure 3: Illustration of the Autler-Townes doublet for different detunings. (a) Two-dimensional plot with the MW frequencies on the $\mathrm{x}$-axis and the RF detuning on the y-axis for menthone (conformer $\mathrm{A}$ ). (b) Spectra of different detunings close to $\Delta_{R F}=0$. Note the intensities of the doublet are equal for zero detuning.

\section{References}

[1] Karl Blum. Density Matrix Theory and Applications. Springer-Verlag, 3rd ed. edition, 2011. 